\title{
Baixo peso ao nascer e fatores associados ao pré-natal: estudo seccional em uma maternidade de referência de Macaé
}

\section{Jane de Carlos Santana Capelli}

Universidade Federal do Rio de Janeiro Campus

UFRJ-Macaé Professor Aloisio Teixeira.

\section{Camilla Medeiros Macedo da Rocha}

Universidade Federal do Rio de Janeiro Campus UFRJ-Macaé Professor Aloisio Teixeira.

\section{Luana Silva Monteiro}

Universidade Federal do Rio de Janeiro Campus UFRJ-Macaé Professor Aloisio Teixeira.

\section{Naiara Sperandio}

Universidade Federal do Rio de Janeiro Campus UFRJ-Macaé Professor Aloisio Teixeira.

\section{Alexand ra da Silva Anastacio}

Universidade Federal Fluminense.

\section{Silvia Pereira}

Universidade Federal Fluminense.

\section{Cleber Nascimento do Carmo}

Escola Nacional de Saúde Pública/Fundação Oswaldo Cruz.

\section{Maria Fernanda Larcher de Almeida}

Universidade Federal do Rio de Janeiro Campus UFRJ-Macaé Professor Aloisio Teixeira.

\section{Resumo}

Objetivos: Estimar a associação entre o baixo peso ao nascer (BPN) e os fatores socioeconômicos, demográficos e assistência pré-natal de puérperas atendidas em uma maternidade de referência de Macaé. Métodos: Realizou-se um estudo seccional, descritivo, de bases primária e secundária na maternidade de um hospital público do município, entre agosto e dezembro de 2014. Realizou-se uma análise múltipla de correspondências para descrição e elaboração de perfil multivariado entre as variáveis socioeconômicas, demográficas e assistência pré-natal com o BPN. Resultados: Foram entrevistadas 105 puérperas, com idade média de 25,5 anos e observou-se $8,6 \%$ de BPN. O perfil multivariado sinalizou que recémnascidos com BPN tendem a ser aqueles cuja mãe apresentou baixo peso pré-gestacional, renda inferior a um salário mínimo, realizou menos de 6 consultas de pré-natal e idade gestacional a termo. Conclusão: O BPN foi associado ao baixo peso pré-gestacional materno, baixa renda familiar (inferior a 1 salário mínimo) e o número de consultas prénatal $(<6)$.

Palavras-chave: Baixo Peso ao Nascer; Saúde da Criança; Maternidade.

\section{Abstract}

Objectives: To estimate the association between low birth weight (LBW) and socioeconomic, demographic factors and prenatal care of postpartum women attended in a reference maternity in Macaé. Methods: A descriptive, sectional study of primary 
and secondary bases in the maternity hospital of a public hospital in the municipality was carried out between August and December 2014. A multiple correspondence analysis was performed to describe and elaborate a multivariate profile among socioeconomic variables, and prenatal care with LBW. Results: 105 postpartum women were interviewed, with an average age of 25.5 years. LBW was $8.6 \%$. In the first group, it was detected that newborns with LBW tend to be those whose mothers

\section{Introdução}

O baixo peso ao nascer (BPN) é considerado o principal fator de risco para a sobrevivência do recém-nascido ${ }^{1}$, estando associado a morbimortalidade perinatal quando avaliada a gravidez como desfecho, bem como às condições socioeconômicas de um país ${ }^{2}$, além de ser o indicador isolado relacionado à mortalidade infantil ${ }^{3,4}$ e preditor da qualidade de vida do ser humano. ${ }^{5}$

O BPN pode ser um reflexo do estado nutricional da gestante e do recém-nascido, influenciando diretamente no crescimento e desenvolvimento do ser humano desde o nascimento até nas condições de saúde na fase adulta. ${ }^{6,7}$

A Organização Mundial da Saúde (OMS) define o BPN como o peso ao nascer inferior a $2.500 \mathrm{~g}^{1,8}$, tendo como principais responsáveis por esta condição: o retardo do crescimento intrauterino (RCIU) e a prematuridade, ou seja, idade gestacional inferior a 37 semanas. ${ }^{9}$ Além desses principais fatores a literatura também destaca partos múltiplos, had low pre-gestational weight, income less than a minimum wage, performed less than 6 prenatal consultations and full term gestational age. Conclusion: The LBW was associated with low maternal pre-gestational weight, low family income (less than 1 minimum wage) and the number of prenatal consultations $(<6)$.

Keywords: Low Birth Weight; Child Health; Maternity.

hipertensão arterial, baixo índice de massa corporal e baixa estatura materna, tabagismo e baixo ganho de peso durante a gravidez, primiparidade, anomalias congênitas e fatores genéticos. ${ }^{10}$

Estima que o BPN atinja cerca de $15,0 \%$ a $20,0 \%$ de todos os nascimentos no mundo, representando mais de 20 milhões de nascimentos, tendo como meta a redução em $30,0 \%$ dos recém-nascidos com BPN até o ano 2025. Assim, espera-se uma redução relativa de 3,0\% por ano entre 2012 e 2025 e uma redução de aproximadamente 20 milhões para cerca de 14 milhões de crianças com BPN. ${ }^{1,11}$ No Brasil, no ano de 2012, o BPN foi estimado em $8,5 \%$ de todos os nascimentos, apresentando pequenas variações por região, de 9,2\% na região Sudeste a 7,5\% na região Norte. ${ }^{12}$

Neste sentido, o presente estudo tem o objetivo estimar a associação entre o BPN e fatores socioeconômicos, demográfico e prénatal de puérperas atendidas em uma maternidade de referência de Macaé. 


\section{Métodos}

Trata-se de um estudo seccional, descritivo, quantitativo, com coleta de dados primários e secundários hospitalares de puérperas adultas, moradoras de Macaé-RJ, atendidas em uma maternidade de referência do município, desenvolvido entre agosto e dezembro de 2014.

Este trabalho faz parte do projeto de pesquisa matriz intitulado como "Fatores demográficos, socioeconômicos, assistência pré-natal e nutricional relacionados ao baixo peso ao nascer: um estudo transversal realizado em um hospital municipal de Macaé, RJ", que está em atendimento à Resolução do Conselho Nacional de Saúde (CNS) no 466, de 12 de dezembro de 2012, sendo aprovado pelo Comitê de Ética em Pesquisa da Faculdade de Medicina de Campos dos Goytacazes-RJ, com o CAAE no 32809614.1.0000.5244.

O município de Macaé localiza-se na região Norte Fluminense, com estimativa populacional de 206.728 habitantes $^{13}$ e registro de 4.174 nascidos vivos filhos de mães residentes no município (Sinasc/DataSUS) para o ano de 2014. ${ }^{14}$ A maternidade que foi locus do estudo registrou 2.432 bebês nascidos vivos em 2014 .

Os critérios de elegibilidade das puérperas (e seus recém-nascidos) foram: ser residente no município de Macaé, terem à época do estudo idade entre 20 e 34 anos. Os critérios de exclusão adotados foram: registro de complicações no parto, gestação de alto risco (com doença obstétrica na gestação atual, tais como exemplo de pré-eclâmpsia, eclâmpsia, e diabetes gestacional), recém-nascidos prematuros com baixo peso ou peso insuficiente para a idade gestacional (para evitar vieses).

O tamanho amostral foi calculado tendo em vista uma população finita de 2500 partos por ano, com prevalência estimada para o baixo peso de $10 \%$, margem de erro de $5 \%$, intervalo de confiança de $90 \%$, consideraram-se $10 \%$ a mais para eventuais perdas, totalizando 103 puérperas.

A coleta de dados foi realizada por um entrevistador, previamente treinado para aplicação do questionário, no período entre agosto e dezembro de 2014, em três turnos na semana, que entrevistou as puérperas nas primeiras 48 horas após admissão no alojamento conjunto, na maternidade do Hospital Público de Macaé (HPM). Os dados antropométricos e aqueles desconhecidos pelas puérperas foram coletados nos prontuários médicos ou no cartão da gestante. As puérperas foram esclarecidas sobre o estudo e assinaram do Termo de Consentimento Livre e Esclarecido. Não houve recusas no decorrer da coleta.

O questionário da pesquisa foi um instrumento estruturado com informações sobre a puérpera e seu recém-nascido, a partir das seguintes variáveis: (a) dados demográficos - idade materna, (b) dados socioeconômicos - estado civil [tem companheiro e não tem companheiro], escolaridade (anos de estudo) e 
renda familiar ( $\mathrm{R} \$$ ); (c) dados antropométricosestatura $(\mathrm{m})$, peso pré-gestacional $(\mathrm{Kg})$, índice de massa corporal pré-gestacional $\left(\mathrm{Kg} / \mathrm{m}^{2}\right)$, peso ao nascer em gramas do recém-nascido; (d) outras variáveis - idade gestacional (em semanas) na primeira consulta, classificação nutricional pré-gestacional, classificação do peso ao nascer e frequência ao pré-natal (número de consultas). A medida da idade gestacional foi baseada na data da última menstruação. As informações desconhecidas pelas puérperas foram obtidas nos prontuários da maternidade e no cartão da gestante, fontes aqui compreendidas como secundárias.

A classificação das variáveis antropométricas se deu a partir do estado nutricional prégestacional, determinado pelo Índice de Massa Corporal (IMC), obtido pela relação peso $(\mathrm{Kg}) /$ estatura $(\mathrm{m})^{2}$; com classificação pelos pontos de corte do National Academy of Sciences do Institute of Medicine ${ }^{15}$, no qual o IMC $<18,5$ = baixo peso; entre 18,5 - 24,9 é peso adequado; entre $25,0-29,9$ classifica como sobrepeso; e $\geq 30,0$ obesidade.

Para a classificação do peso ao nascer seguiu-se o critério recomendado pela $\mathrm{OMS}^{16}$, com os pontos de corte de: Baixo peso: <2500g; Peso insuficiente: 2500 a 2999g; Peso adequado: 3000 a 3999g; Excesso de peso: $\geq 4000$ g.

Os dados foram digitados e consolidados utilizando-se os softwares Excel for Windows 2007 e o SPSS/PC, versão 19.0. Para a análise estatística foram calculadas, inicialmente, estatísticas descritivas para caracterização da amostra. Posteriormente, foi realizada uma descrição bivariada das características socioeconômicas, demográficas e de pré-natal das puérperas, comparando as proporções através do teste $\chi^{2}$, considerando um nível de significância de 5\%. Finalmente, buscou-se explorar relações conjuntas entre os fatores socioeconômicos, demográficos e de pré-natal das puérperas por meio da análise de correspondência múltipla.

A análise de correspondência é uma técnica exploratória de estatística multivariada utilizada para verificar associações ou similaridades entre variáveis categóricas ou categorizadas. É uma ferramenta indicada no estudo de fatores de risco potencialmente associados a determinadas características que se deseja investigar, bem como permitiria a identificação de grupos com mesmos fatores de risco.

O principal resultado da técnica é uma representação gráfica em que as posições das categorias de cada variável no plano multidimensional podem ser interpretadas como associações. Quanto maior a proximidade entre os pontos, maior a associação entre as categorias ali exibidas. Um conjunto de eixos fatoriais é obtido de modo a maximizar a variabilidade explicada dos dados, explicando, assim, a configuração das categorias das variáveis. A qualidade da análise é mensurada pela inércia relativa que, quanto maior o seu valor, melhor é o ajuste obtido. 
Foram consideradas nesta análise: peso ao nascer (insuficiente; baixo peso; peso adequado; excesso de peso); IMC prégestacional (baixo peso, peso adequado, sobrepeso e obesidade); escolaridade materna ( $<9$ anos ou até 8 anos inclusive; $\geq 9$ anos ou mais de 8 anos); estado civil (sem companheiro; com companheiro); idade gestacional do recém-nascido (pré-termo; a termo); número de consultas pré-natal $(<6 ; \geq 6)$; renda familiar em salários mínimos $(<1 ; 1-2 ; 3-4 ; \geq 5)$.

\section{Resultados}

A amostra total do estudo foi composta por 105 puérperas. A idade materna apresentou média de $25,5 \pm 4,01$ anos e a idade gestacional em semanas, na primeira consulta, de $13,9 \pm 5,68$. A estatura média foi de $1,59 \pm 0,06$ metros e IMC pré-gestacional de $24,82 \pm 4,93 \mathrm{~kg} \cdot \mathrm{m}^{2}$. O peso ao nascer apresentou média de 3193 gramas, com valores mínimo e máximo variando de 1150 a 4285 gramas (Tabela 1$)$.

Tabela 1. Valores médios, desvio padrão das variáveis demográficas, antropométricas e peso ao nascer de usuários da maternidade do Hospital Público de Macaé-RJ (HPM), entre agosto e dezembro/2014. $(n=105)$

\begin{tabular}{|l|c|c|c|}
\hline \multicolumn{1}{|c|}{ Variáveis } & Média & Mínimo & Máximo \\
\hline Idade materna (anos) & $25,5 \pm 4,01$ & 20 & 34 \\
\hline Idade gestacional (semanas) & $13,9 \pm 5,68$ & 5 & 40 \\
\hline Peso Pré-Gestacional (kg) & $62,8 \pm 13,18$ & 38 & 94 \\
\hline Estatura (m) & $1,59 \pm 0,06$ & 1,46 & 1,76 \\
\hline IMC Pré-Gestacional & $24,82 \pm 4,93$ & 38,14 & 17,2 \\
\hline Peso ao Nascer do $\mathrm{RN}^{3}(\mathrm{~g})$ & $3193,8 \pm 568,04$ & 1150 & 4285 \\
\hline
\end{tabular}

Legenda: ${ }^{1}$ na primeira consulta.

'Índice de Massa Corporal Pré-Gestacional.

${ }^{3} \mathrm{RN}$ : Recém-Nascido.

Em relação a s características socioeconômicas, demográficas e de assistência pré-natal, observou-se uma maior prevalência de puérperas na faixa etária de 20 a 25 anos $(52,4 \%)$, baixa escolaridade (91,3\%), renda familiar igual ou menor a dois salários mínimos (64,7\%), número de consultas pré-natal igual ou maior $6(67,6 \%)$, e maior prevalência $(49,9 \%)$ de
IMC pré-gestacional adequado, no entanto, pode-se destacar a prevalência expressiva $(39,7 \%)$ de puérperas com excesso de peso (sobrepeso mais obesidade). Em relação ao recém-nascido, $88,6 \%$ nasceram a termo e $64,8 \%$ com peso adequado ao nascer. O BPN foi de $8,6 \%$ e, somando-se ao peso insuficiente, apresentaram a prevalência de 29,6\% (Tabela 2). 
Tabela 2. Proporções das variáveis socioeconômicas, demográficas e assistência pré-natal, de puérperas atendidas no Hospital Municipal Público de Macaé (HPM), entre agosto e dezembro/2014. $(n=105)$

\begin{tabular}{|c|c|c|}
\hline Variáveis & $N(\%)$ & p-valor ${ }^{1}$ \\
\hline \multicolumn{3}{|l|}{ Idade (anos) } \\
\hline $20-25$ & $55(52,4)$ & $<0,01$ \\
\hline $25-30$ & $34(32,4)$ & \\
\hline $30-35$ & $16(15,2)$ & \\
\hline \multicolumn{3}{|l|}{ Estado Civil } \\
\hline Com companheiro & $62(59,6)$ & 0,05 \\
\hline Sem companheiro & $42(40,4)$ & \\
\hline \multicolumn{3}{|l|}{ Escolaridade (anos) } \\
\hline$<9$ & $95(91,3)$ & $<0,01$ \\
\hline$\geqslant 9$ & $9(8,7)$ & \\
\hline \multicolumn{3}{|l|}{ Renda Familiar (SM)² } \\
\hline$<1$ & $4(4,7)$ & $<0,01$ \\
\hline $1-2$ & $51(60,0)$ & \\
\hline $3-4$ & $22(25,9)$ & \\
\hline$\geqslant 5$ & $8(9,4)$ & \\
\hline \multicolumn{3}{|c|}{ № consultas pré-natal } \\
\hline$<6$ & $34(32,4$ & $<0,01$ \\
\hline$\geqslant 6$ & $71(67,6)$ & \\
\hline \multicolumn{3}{|l|}{ IMC Pré-gestacional $\left.\right|^{3}$} \\
\hline Baixo Peso & $11(10,9)$ & $<0,01$ \\
\hline Peso Adequado & $50(49,9)$ & \\
\hline Sobrepeso & $15(14,9)$ & \\
\hline Obesidade & $25(24,8)$ & \\
\hline \multicolumn{3}{|c|}{ Idade gestacional do RN } \\
\hline Pré-termo & $12(11,4)$ & $<0,01$ \\
\hline A termo & $93(88,6)$ & \\
\hline \multicolumn{3}{|l|}{ Peso ao nascer (g) } \\
\hline Baixo peso & $9(8,6)$ & $<0,01$ \\
\hline Peso insuficiente & $22(21,0$ & \\
\hline Peso adequado & $68(64,8)$ & \\
\hline Excesso de peso & $6(5,7)$ & \\
\hline
\end{tabular}

Legenda: ${ }^{1}$ Teste qui-quadrado não paramétrico.

${ }^{2} \mathrm{SM}$ : Salário Mínimo.

${ }^{3}$ Institute of Medicine (2009). 
A Figura 1 apresenta um perfil multivariado das associações de BPN com as variáveis socioeconômicas, demográficas e de assistência pré-natal das puérperas do estudo, sendo possível a identificação de quatro grupos. A inércia relativa da análise de correspondências foi capaz de explicar 50,5\% da variabilidade total das variáveis nos dois eixos do gráfico.

Observou-se que recém-nascidos com BPN, tendem a ser aqueles em que a mãe apresentava também baixo peso pré-gestacional, renda inferior a um salário mínimo, realizou menos de 6 consultas de pré-natal ao longo da gestação e idade gestacional a termo. Num segundo grupo, é possível identificar puérperas com sobrepeso pré-gestacional, cujos recém-nascidos apresentaram excesso de peso ao nascer e com renda entre 3 e 4 salários mínimos. Um terceiro grupo é visto com puérperas sem companheiro, renda entre 1 e 2 salários mínimos, peso insuficiente ao nascer do recém-nascido, porém com puérpera com peso pré-gestacional adequado. Finalmente, o quarto grupo é caracterizado por puérperas com companheiro, renda superior a 5 salários mínimos, obesidade pré-gestacional, mais que 6 consultas de prénatal realizadas e recém-nascidos com peso adequado no momento do nascimento (Figura 1).

Figura 1. Resultado da análise de correspondências múltiplas de baixo peso ao nascer com variáveis socioeconômicas, demográficas e assistência pré-natal, de puérperas atendidas no Hospital Municipal Público de Macaé (HPM), entre agosto e dezembro/2014. ( $n=105)$

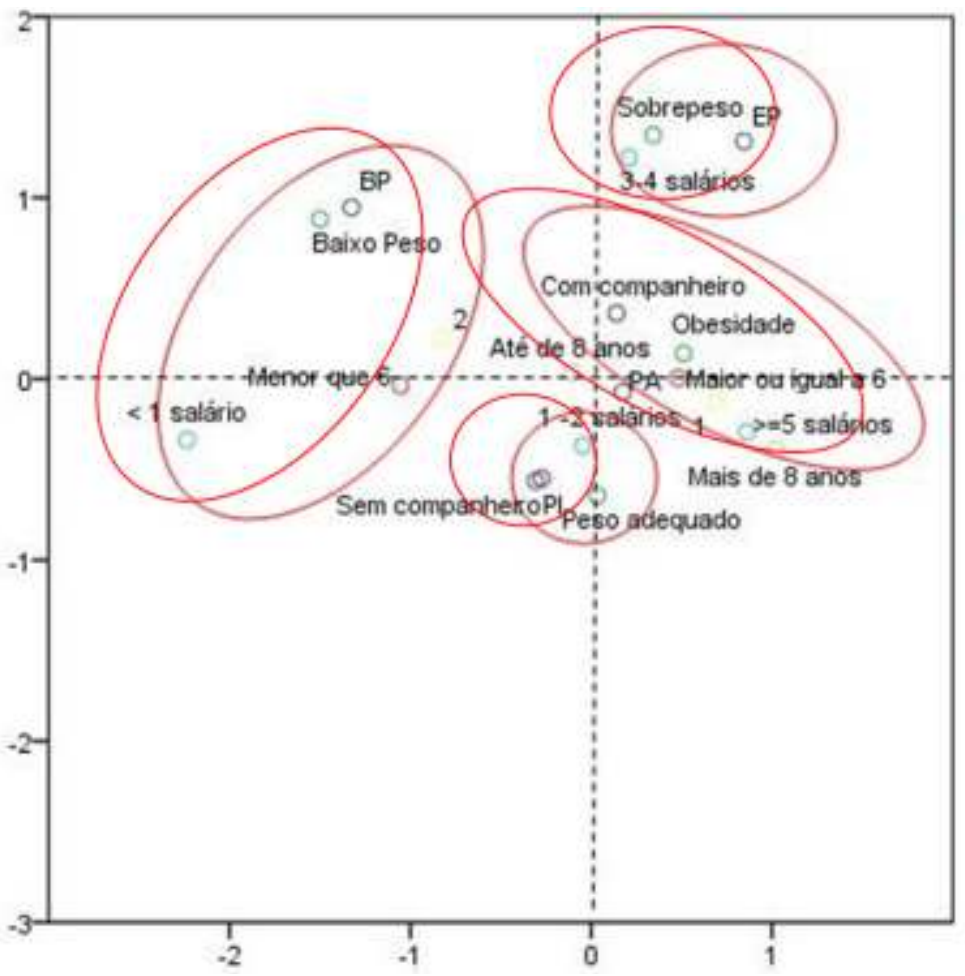

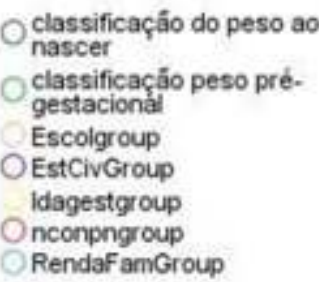

PI: Peso Insuficiente; BP: Baixo Peso; PA: Peso Adequado; EP: Excesso de Peso (classificação do peso ao nascer).

Baixo Peso, Peso Adequado Sobrepeso e Obesidade (classificação do Índice de Massa Corporal PréGestacional = classificação peso pré-gestacional). Mais de 8 anos ou $\geq 9$; Até 8 anos ou $<9$ ) (Escolaridade materna $=$ Escolgroup) .

Sem companheiro; com companheiro (Estado civil = EstCivGroup).

1: pré-termo; 2: a termo (Idade gestacional do recémnascido $=$ Idagestgroup).

Menor que 6; Maior ou igual a 6 (número de consultas pré-natal $=$ nconpngroup).

$\langle 1 ; 1-2 ; 3-4 ;>=5$ (Renda familiar = RendaFamGroup). 


\section{Discussão}

Os fatores associados ao BPN no presente estudo foram o baixo peso pré-gestacional materno, baixa renda familiar (menor que 1 salário mínimo) e o número de consultas prénatal inferior a seis. Em direção oposta, os fatores associados ao excesso de peso ao nascer foram o sobrepeso pré-gestacional e renda entre 3 e 4 salários mínimos.

A prevalência de BPN encontrada foi próxima ao estimado para o Brasil (8,5\%), no ano de 2012, de todos os nascimentos. ${ }^{12}$ Essa prevalência é preocupante, uma vez que o BPN é considerado preditor da qualidade de vida do indivíduo, estando relacionado ao déficit de desenvolvimento e maior mortalidade infantil. Aponta-se que crianças com BPN apresentam 20 vezes mais chances de morrer em comparação aquelas com peso normal. ${ }^{17}$

Pesquisas conduzidas em diferentes locais ${ }^{18,19}$ observaram resultados semelhantes aos do presente estudo, particularmente com relação a associação do baixo peso pré-gestacional materno, baixa renda familiar e o acompanhamento de pré-natal com a ocorrência do BPN.

O baixo nível socioeconômico se constitui em um importante fator de risco para o BPN, visto que está relacionado a uma precária assistência pré-natal. Estudos envolvendo diferentes regiões do Brasil mostraram que mães que pertencem a grupos socialmente menos favorecidos recebem assistência pré-natal de pior qualidade. ${ }^{20,21}$ Além disso, a baixa renda está relacionada a piores condições de saneamento básico, alimentação e moradia, que também se relacionam a desfechos insatisfatórios de peso ao nascer. ${ }^{19}$

No Brasil, cabe destacar a distribuição espacial desigual das taxas de BPN, refletindo assim as condições socioeconômicas dos estados brasileiros. De acordo com Lima e colaboradores $^{22}$, as regiões mais desenvolvidas do país, Sul e Sudeste, apresentam as maiores taxas de BPN, sendo esse fenômeno conhecido como paradoxo do BPN. ${ }^{23}$ Esse fenômeno é explicado pela presença de serviços e sua utilização pela população, contribuindo para redução da mortalidade infantil e aumento do BPN. Assim, nas áreas menos desenvolvidas, a atenção inadequada e a precariedade de acesso à tecnologia perinatal, contribui para o aumento da taxa de mortalidade infantil. ${ }^{22}$

A precariedade da assistência pré-natal também está relacionada a outro fator que foi determinante, no presente estudo, ao BPN, que se refere ao baixo número de consultas prénatal. De acordo com o Ministério da Saúde, a primeira consulta de pré-natal deve acontecer até o 4o mês de gestação, devendo ser no mínimo 6 consultas ao longo da gestação. ${ }^{24} \mathrm{O}$ cumprimento desta agenda garante uma assistência pré-natal adequada que contribui favoravelmente a saúde materna e infantil. Entretanto, deve-se refletir se a agenda está sendo cumprida e qual a qualidade dessa 
assistência pré-natal. Desta forma, todas as gestantes e recém-nascidos precisam de cuidados especializados e que ocorram em um ambiente humanizado. A qualidade adequada do atendimento requer o uso apropriado de métodos clínicos e não clínicos, infraestrutura dos serviços e melhores aptidões e atitudes dos profissionais de saúde. Buscando assim, melhores resultados de saúde e experiência positiva. Além disso, a qualidade do atendimento é considerada um ponto chave do direito à saúde, e o caminho a equidade e dignidade para mulheres e crianças. De acordo com a OMS, os cuidados de saúde precisam ser seguros, eficazes, oportunos, eficiente, equitativo e centrado nas pessoas. ${ }^{25}$

O baixo peso pré-gestacional foi associado ao BPN no presente estudo. $O$ estado nutricional pré-gestacional é importante prognóstico da gestação. Alguns estudos apontam que mulheres que iniciaram a gravidez com menos de $50 \mathrm{~kg}$ apresentaram maior risco de te filhos com BPN. ${ }^{26,27}$

Outros fatores, que apesar de não terem apresentado significância estatística e/ou não foram investigados neste estudo, mas que são frequentemente associados ao BPN na literatura, e que merecem destaque nas ações de cuidado materno-infantil, são a prematuridade, gestações múltiplas, sexo do feto e exposição a agrotóxicos. ${ }^{28} \mathrm{O}$ direito universal à saúde garantida constitucionalmente no Brasil deve estar atrelado a uma assistência ao grupo materno-infantil de qualidade, através da oferta de serviços públicos de qualidade para toda a população. ${ }^{29}$

Diante desse cenário, a Estratégia Global para a Saúde das Mulheres, das Crianças e dos Adolescentes (2016-2030), busca garantir para essa população seu direito à saúde física e mental e ao bem-estar, tendo oportunidades sociais e econômicas, e que possam participar plenamente da construção de sociedades prósperas e sustentáveis. ${ }^{30}$ Para alcançar, tais propósitos, a estratégia apresenta objetivos e metas alinhados com os objetivos de desenvolvimento sustentável, como sobreviver (acabar com as mortes evitáveis), prosperar (assegurar a saúde e o bem-estar) e transformar (ampliar ambientes propícios). ${ }^{30}$

As limitações do estudo incluem a possível subnotificação de informações, devido à ausência de dados nos prontuários ou a dificuldade de relato pelas gestantes durante a entrevista. Adicionalmente, o desenvolvimento da pesquisa em apenas uma maternidade do município de Macaé-RJ não permite que os achados deste estudo sejam generalizados. No entanto, é importante ressaltar que a maternidade onde o estudo foi realizado é a unidade de referência da região, registrando 2.432 bebês nascidos vivos em 2014 .

\section{Considerações Finais}

A prevalência do BPN foi muito próxima a nacional e apresentou associação com o baixo peso pré-gestacional materno, baixa renda 
familiar (menor que 1 salário mínimo) e o número de consultas pré-natal inferior a seis.

\section{Como são escassos estudos voltados a saúde materno infantil no município de Macaé-RJ, a presente pesquisa propiciará o conhecimento da realidade local para que seja possível}

contribuir com os gestores e profissionais de saúde de modo a traçarem a intervenção no período pré-natal em seu tempo oportuno, em consonância às Políticas de Atenção Integral à Saúde da Mulher e da Criança e aos princípios norteadores do Sistema Único de Saúde(SUS).

\section{Referências}

1. World Health Organization. Global targets 2025. To improve maternal, infant and young child nutrition. [Acessed 17 Jun 2018]. Available: http://www.who.int/nutrition/topics/nutrition_globaltargets2025/en/.

2. Demitto MO, Gravena AAF, Dell'Agnolo CM, Antunes MB, Pelloso SM. High risk pregnancies and factors associated with neonatal death. Rev Esc Enferm USP. 2017; 51:e03208.

3. Capelli JCS, Pontes JS, Pereira SEA, Silva, AAM, Carmo CN, Boccolini CS, Almeida MFL. Peso ao nascer e fatores associados ao período pré-natal: um estudo transversal em hospital maternidade de referência. Cienc Saude Coletiva. 2014; 19(7):2063-2072.

${ }^{4}$. Kramer MS. Determinants of low birth weight: methodological assessment and meta-analysis. Bull World Health Organ. 1987; 65(5):663-737.

5. Lima GSP, Sampaio HAC. Influência de fatores obstétricos, socioeconômicos e nutricionais da gestante sobre o peso do recém-nascido: estudo realizado em uma maternidade em Teresina, Piauí. Rev Bras Saúde Matern Infant, Recife. 2004; 4(3):253-261.

6. Tourinho AB, Reis LBSM. Peso ao Nascer: Uma Abordagem Nutricional. Com Cienc Saude. 2013; 22(4):19-30.

7. Ferraz TR, Neves ET. Fatores de risco para baixo peso ao nascer em maternidades públicas: um estudo transversal. Rev Gaúcha Enferm. 2011; 32(1):86-92.

${ }^{8}$. Resolution WHA65.6. Comprehensive implementation plan on maternal, infant and young child nutrition. In: Sixty-fifth World Health Assembly Geneva, 21-26 May 2012. Resolutions and decisions, annexes. Geneva: World Health Organization; 2012:12-13 [Acessed 17 Jun 2018]. Available: http://www.who.int/nutrition/ topics/WHA65.6_resolution_en.pdf?ua=1.

9. Mendes CQS, Cacella BCA, Mandetta MA, Balieiro MMFG. Low birth weight in a municipality in the southeast region of Brazil. Rev Bras Enferm. 2015;68(6):857-63

${ }^{10}$. Carniel EF, Zanolli ML, Antônio MARGM, Morcillo AM. Determinantes do baixo peso ao nascer a partir das Declarações de Nascidos Vivos. Rev Bras Epidemiol. 2008; 11(1):169-179.

11. WHO/NMH/NHD/14.5. Global Nutrition Targets 2025 Low Birth Weight Policy Brief. [Acessed Jun 26 2018]. Disponível em: http://apps.who.int/iris/bitstream/10665/149020/2/WHO_NMH_NHD_14.5_eng.pdf?ua=1.

12. Brasil. Ministério da Saúde. Secretaria de Vigilância em Saúde. Departamento de Análise de Situação em Saúde. Saúde Brasil 2013: uma análise da situação de saúde e das doenças transmissíveis relacionadas à pobreza/Ministério da Saúde, Secretaria de Vigilância em Saúde, Departamento de Análise de Situação em Saúde. - Brasília: Ministério da Saúde, 2014. 384 p.: il.

13. Fundação Instituto Brasileiro de Geografia e Estatística. Macaé. Rio de Janeiro. Cidades [Internet]. Rio de Janeiro: Instituto Brasileiro de Geografia e Estatística; 2016. [Acesso em 6 Nov 2017]. Disponível em: http://cidades.ibge.gov.br/xtras/perfil. php?codmun=330240.

${ }^{14}$. Ministério da Saúde (BR). Departamento de Informática do SUS - DATASUS/Estatísticas Vitais. Sistema de Informações sobre Nascidos Vivos - SINASC, 2014 . [Acesso 10 Set 2017]. Disponível em: http://tabnet.datasus.gov.br/cgi/tabcgi.exe?sinasc/cnv/nvrj.def. 
${ }^{15}$. Institute of Medicine. National Research Council. Weight Gain During Pregnancy: Reexamining the Guidelines. Washington (DC): National Academy of Science, 2009.

16. Puffer RR, Serrano C. Patterns of birth weight. PAHO Scientific Publication no 504, Washington, DC: PAHO, 1987.

17. World Health Organization (WHO), United Nations Children's Fund (Unicef). Low birthweiight: contry, regional and global estimates. New York: WHO, Unicef; 2004.

18. Sally EOD, Werneck GL. Desigualdade econômica e baixo peso ao nascer em Niterói, Rio de Janeiro. Cad Saude Colet. 2010; 18(2):282-90.

19. Vettore MV, Gama SG, Lamarca GA, Schilithz AO, Leal MC. Housing conditions as a social determinant of low birthweight and preterm low birthweight. Rev Saude Publica. 2010; 44(6):1021-31.

20. Coimbra LC, Silva AAM, Mochel EG, Alves MTSSB, Ribeiro VS, Aragão VMF, Bettiol H. Fatores associados à inadequação do uso da assistência pré-natal. Rev Saude Publica 2003; 37(4):456-462.

${ }^{21}$. Gonçalves CV, Cesar JA, Mendoza-Sassi RA. Qualidade e eqüidade na assistência à gestante: um estudo de base populacional no Sul do Brasil. Cad Saude Publica 2009; 25(11):2507-2516.

22. Lima MCBM, Oliveira GS, Lyra CO, Roncalli AG, Ferreira MAF. A desigualdade espacial do baixo peso ao nascer no Brasil. Cienc Saude Coletiva. 2013; 18(8):2443-2452.

${ }^{23}$. Silva AAM, Silva LM, Barbieri MA, Bettiol H, Carvalho LM, Ribeiro VS, Goldani MZ. The epidemiologic paradox of low birth weight in Brazil. Rev Saude Publica. 2010; 44(5):767-775.

${ }^{24}$. Brasil. Ministério da Saúde. Portaria n. 569, de 10 junho 2000. Institui o Programa de Humanização no Pré-Natal e Nascimento. Diário Oficial da União, Brasília (DF) 2000. Jun 8; Sec.1.

25. Ö Tuncalp, WM Were, C MacLennan OT Oladapo, AM G€ulmezoglu, R Bahl, B Daelmans,bM Mathai, L Say, F Kristensen,M Temmerman, F Bustreo. Quality of care for pregnant women and newborns - the WHO vision. BJOG. 2015. 1222(8):1045-9.

${ }^{26}$. Halpern R, Schaefer ES, Pereira AS, Arnt EM, Bezerra JPV, Pinto LS. Fatores de risco para o baixo peso ao nascer em uma comunidade rural do sul do Brasil. J Pediatr. 1996; 72(6):369-73.

${ }^{27}$. Ramakrishman U. Nutrition and low birth weight: from research to practice. Am J Clin Nutr. 2004; 79(1):17-21.

28. Boccolini CS, Carvalho ML, Oliveira MI, Pérez-Escamilla R. Breastfeeding during the first hour of life and neonatal mortality. J Pediatr. 2013; 89(2):131-136.

${ }^{29}$. Machado, CV. O papel federal no sistema de saúde brasileiro. In: Fundação Oswaldo Cruz. A saúde no Brasil em 2030 prospecção estratégica do sistema de saúde brasileiro: organização e gestão do sistema de saúde [online]. Rio de Janeiro: Fiocruz/Ipea/Ministério da Saúde/Secretaria de Assuntos Estratégicos da Presidência da República, 2013. v. 3. 35-70pp.

30. Organização Panamericana de Saúde. Estratégia global para a saúde das mulheres, das crianças e dos adolescentes (2016-2030). [Acesso $17 \mathrm{Jul}$ 2018]. Disponível em: http://www.everywomaneverychild.org/wpcontent/uploads/2017/10/EWEC_Global_Strategy_PT_inside_LogoOK2017_web.pdf. 